\title{
Gamificación y TIC en la formación literaria. Una propuesta didáctica innovadora en Educación Secundaria
}

\author{
Alícia Martí Climent ${ }^{1}$; Pilar Garcia Vidal ${ }^{2}$
}

Recibido: 30 de septiembre de 2020 / Aceptado: 14 de julio de 2021

Resumen. La gamificación en la formación literaria puede aportar una serie de ventajas en su aplicación didáctica de manera que repercuta en un aprendizaje significativo en el aula. Los participantes en el juego de la experiencia que presentamos, organizados en grupos, tienen que superar un reto mediante la resolución de una serie de enigmas o pruebas relacionadas antes de que se acabe el tiempo establecido. De esta forma, el alumnado lee, interpreta, dialoga, opina, escribe y crea para poder conseguir el objetivo de resolver la situación planteada. Los recursos digitales y la lectura comprensiva permiten que los enigmas se resuelvan mediante el trabajo colaborativo y se consiga elaborar un producto final original que se puede compartir y difundir fácilmente.

Palabras clave: Gamificación en educación; TIC; Literatura; Innovación educativa.

\section{[en] Gamification and ICT in literacy training. An innovative didactic proposal in Secondary School}

Abstract. Gamification in literacy training may provide a serie of advantages in its didactic implementation so that it makes an impact in a meaningful learning in the classroom. Participants enrolled in teams, taking part in this work experience, have to achieve a challenge by solving a serie of mysteries or related tasks before the set time. In this way, students read, perform, dialog, write and create in order to achieve the aim and solve the situation. Through the digital resources and comprehension reading the mystery can be solved, taking always into account the collaborative work. An original final product can be produced to be shared and disseminated easily.

Keywords: Gamification in education; ICT; Literature; Educational innovations.

[fr] Gamification et TIC dans la formation littéraire. Une proposition didactique innovante dans l'enseignement secondaire

Résumé. La gamification dans la formation littéraire peut apporter une série d'avantages dans son application didactique de manière à ce qu'elle répercute sur un apprentissage significatif en classe. Les participants au jeu de l'expérience que nous présentons, organisés en groupes, doivent surmonter un défi en résolvant une série d'énigmes ou d'épreuves liées entre elles avant que le temps établi finisse. De cette façon, les élèves lisent, interprètent, dialoguent, jugent, écrivent et créent pour pouvoir atteindre leur objectif et donc résoudre la situation proposée. Les ressources numériques et la lecture compréhensive permettent de résoudre les énigmes moyennant le travail collaboratif et d'obtenir un produit final original qui peut être facilement partagé et diffusé.

Mots-clés: Gamification dans l'éducation; TIC; Littérature; Innovation éducative.

Sumario: 1. Introducción. 2. Fundamentación teórica. 2.1. La gamificación en educación. 2.2. Las TIC y la gamificación. 2.3. Escape room en Educación Secundaria. 3. Propuesta didáctica. 3.1. Objetivos. 3.2. Contenidos y competencias. 3.3. Metodología. 3.4. Actividades. 3.5. Recursos. 3.6. Evaluación. 4. Resultados. 5. Conclusiones. 6. Referencias bibliográficas.

Cómo citar: Martí Climent, Alícia y Pilar García Vidal (2021). Gamificación y TIC en la formación literaria. Una propuesta didáctica innovadora en Educación Secundaria. Didáctica. Lengua y literatura, 33, 109-120. 


\section{INTRODUCCIÓN}

Con este trabajo queremos poner en evidencia que, aunque muchas veces se ha querido enfrentar los libros y la tecnología, podemos trabajarlos conjuntamente y con un buen resultado mediante la gamificación. Se trata de promover estrategias válidas para la educación literaria de manera que el alumnado aprenda leyendo, hablando y escribiendo sobre lo que lee.

De forma similar, tradicionalmente los juegos han sido considerados la antítesis del aprendizaje, aunque durante los últimos años se ha demostrado la conexión existente entre estos y el aprendizaje. Así lo constatan en la revisión de la literatura sobre gamificación educativa Parra-González y Segura-Robles (2019, p. 117) cuando destacan que "la introducción de la mecánica de los juegos con un objetivo concreto en las aulas proporciona aprendizajes significativos y que el uso de los mismos ha demostrado proporcionar un incremento del aprendizaje (De-Marcos, Domínguez, Sáenz-de-Navarrete y Pagés, 2014; Gee, 2007)".

De acuerdo con Corchuelo-Rodríguez (2018), las nuevas tendencias educativas integran metodologías activas y participativas donde los estudiantes adquieren una postura dinámica en su aprendizaje. En este sentido, la gamificación emerge como una herramienta de transformación educativa.

Además, los estudiantes actuales pertenecen a una generación que maneja las herramientas digitales con mucha soltura, sabe cómo funciona el mundo de las redes sociales y está acostumbrada a realizar simultáneamente varias tareas con el apoyo de la tecnología. Como apuntan Ríos, Muñoz, Castro y Arroyo (2019), varios autores han puesto de manifiesto que la forma en que aprende esta generación está condicionada por su cultura tecnológica y que quieren participar y controlar activamente su formación.

En este artículo presentamos una propuesta didáctica de interés con la que gamificar nuestra aula y crear oportunidades de aprendizaje. Mediante el uso del escape room el alumnado se convierte en protagonista de una historia real de escapismo en la que tiene que mostrar habilidades o manejar conceptos propios de la formación literaria en la Educación Secundaria.

\section{FUNDAMENTACIÓN TEÓRICA}

En los últimos años en el campo científico, según Parra-González y Segura-Robles (2019), están proliferando las investigaciones sobre gamificación educativa. En su análisis cienciométrico de las publicaciones indexadas en las bases de datos de impacto Web of Science (WoS) sobre la metodología emergente de gamificación en Educación, con una muestra compuesta por 345 producciones científicas, se demuestra el incremento del interés de esta metodología en las aulas.

Pero la gamificación o ludificación no es nueva en educación. La utilización del juego como recurso educativo ha existido desde siempre. Piaget (1961) y Vigotsky $(1933,1966)$ ya defendían el uso de elementos del juego en educación como estrategia de aprendizaje, instrumento para el desarrollo mental del niño, destacando que ayuda a crear mundos de imaginación.

Ahora, sin embargo, se plantea desde una perspectiva mucho más global y transformadora: recoger todo el potencial de tiempo y experiencia que el alumnado dedica al juego hacia el aprendizaje. Resulta una estrategia y metodología motivadora para que se dé un proceso de enseñanza-aprendizaje significativo en general. No se trata de diseñar actividades o tareas que se basan en la jugabilidad, sino de "poner el juego en el centro del proceso educativo como una manera de abrir los niños al misterio y la belleza, a la vida" (Marín, 2014).

\subsection{La gamificación en educación}

Contreras y Eguia (2016) apuntan que el concepto de gamification fue definido por Deterding, Dixon, Khaled y Nacke en el artículo "Gamification: Toward a Definition" en 2011 y se refiere al uso de elementos del juego, y el diseño de juegos, para mejorar el compromiso y la motivación de los participantes en contextos que no son de juego: "gamification is the use of game design elements in non-game contexts" (Deterding, Dixon, Khaled y Nacke, 2011, p. 2).

Ortiz-Colón, Jordán y Agredal (2018) realizan una revisión teórica de la producción científica sobre la gamificación en educación desde 2011 hasta 2016, que nos ofrece un estado de la cuestión de los beneficios del uso de la gamificación y de su aplicación en el contexto educativo, y contempla la revisión anterior de Hamari, Koivisto y Sarsa (2014).

De acuerdo con Parente (2016, p. 18), "la gamificación es utilizar mecánicas asociadas al videojuego, para presentar al alumno una serie de retos de aprendizaje, que en cuanto el alumno lo haya cumplido, generará una recompensa a corto plazo dimensionada a la complejidad del reto". Para Ripoll (2016, p. 26), "la gamificación debe poner en el centro al alumno y la forma cómo este percibe los contenidos que se están planteando en clase".

Ramírez (2014) señala que la gamificación tiene entre sus objetivos influir en el comportamiento de las personas, mediante las experiencias y sentimientos que se construyen a través del juego incentivando su compromiso y fidelidad, y, como apunta Teixes (2015), actúa sobre la motivación para la consecución de objetivos concretos. 
Según Contreras y Eguia (2017), Werbach presenta en 2014 una definición muy amplia y general del concepto de gamificación, describiéndolo como un proceso que está destinado a realizar actividades de forma parecida a un juego. En este sentido, Contreras y Eguia (2017, p. 8) proponen que la gamificación podría entenderse (de una forma amplia) como "un proceso en el que el "gamificador" intenta aumentar las probabilidades de la aparición de diferentes experiencias de juego al contagiar posibilidades para ese fin (ya sea mediante distintivos, puntos, etc.)".

Los principios de gamificación descritos por Mark van Diggelen (2012, citado en Parente, 2016, p. 13), sugieren que el proceso de gamificación se puede resumir en 10 puntos:

1. Tipos de competición: jugador versus jugador, jugador versus sistema y/o solo.

2. Presión temporal: jugar de forma relajada o jugar con el tiempo.

3. Escasez: la escasez de determinados elementos puede aumentar al reto y la jugabilidad.

4. Puzles: problemas que indican la existencia de una solución.

5. Novedad: los cambios pueden presentar nuevos retos y nuevas mecánicas que dominar.

6. Niveles y progreso.

7. Presión Social: el rebaño debe saber lo que hace.

8. Trabajo en equipo: puede ser necesario la ayuda de otros para conseguir avanzar.

9. Moneda de cambio: cualquier cosa que puede ser intercambiada por otra de valor, será buscada.

10. Renovar y aumentar poder: permite añadir elementos motivacionales al jugador.

Parente (2016) añade la bidireccionalidad de la interacción y de la relación, ya que considera que el proceso no debe ser una actuación unidireccional, sino que puede darse del profesor en dirección al alumno, de los alumnos al profesor y en ambas direcciones.

Marín $(2015$, p. 1) considera que actualmente podemos hablar de "una línea específica de gamificación educativa, donde los entornos formales introducen recursos propios de los no formales con el fin de potenciar un aprendizaje significativo". Se trata de una tendencia basada en la unión del concepto de ludificación y aprendizaje.

Por otra parte, algunos de los beneficios de la gamificación en educación que señalan Ortiz-Colón, Jordán y Agredal (2018) son la motivación, la inmersión para posibilitar la anticipación y planificación de situaciones; el compromiso y la socialización a través de la interactividad y la interacción; la mejora de la colaboración en el aula; las emociones como elemento favorecedor del proceso de enseñanza/aprendizaje; las actitudes e interacción, y la cooperación entre compañeros. Ortiz-Colón, Jordán y Agredal (2018) destacan la gran influencia que tiene la gamificación en el desarrollo cognitivo de los estudiantes, en las emociones y en los procesos de socialización que se generan a lo largo del proceso.

La gamificación en las aulas es una metodología eficaz, según Contreras (2016, 2017), siempre y cuando se utilice para animar a los estudiantes a progresar a través de los contenidos de aprendizaje, para influir en su comportamiento o acciones y para generar motivación. En relación con la gamificación y la motivación, encontramos también el flow (Csikszentmihalyi, 1975, 1990), es decir el estado mental que alcanza una persona cuando está totalmente inmersa y concentrada en la actividad que está realizando, de modo que pierde la noción del tiempo porque está absorta en aquello que hace ya que le genera satisfacción.

Werbach y Hunter (2012), citados en Corchuelo-Rodríguez (2018), consideran que para implementar una estrategia de gamificación exitosa es necesario tener en cuenta seis elementos:

1. Definir con claridad los objetivos educativos que se quieren conseguir.

2. Delimitar los comportamientos que queremos potenciar en los estudiantes.

3. Establecer quiénes son los jugadores, identificar rasgos y características.

4. Establecer los ciclos de las actividades (mecánicas de juego, orden de los eventos, interacción, entre otros).

5. Diversión (es la base de todo juego).

6. Recursos, incluye las herramientas que se van a usar para el desarrollo de la estrategia.

Contreras y Eguia recogen una muestra de experiencias desarrolladas en las aulas universitarias (2016) y también posteriormente en las aulas de primaria y secundaria (2017).

Según Contreras y Eguia (2016), algunos profesores universitarios utilizan la gamificación para alentar a los estudiantes a que se involucren en las asignaturas, para motivar la acción, influir en su comportamiento, fomentar una competencia amigable entre los estudiantes, mejorar habilidades, el proceso de evaluación y la adquisición de conocimientos.

Paula (2017) presenta una experiencia de gamificación en $3^{\circ}$ de Educación Primaria para mejorar la competencia lectora y animar a la lectura. En este caso se entiende la gamificación como la aplicación de reglas y mecánicas relacionadas con el juego en una determinada tarea. Se propone al alumnado una serie de retos u objetivos finales y durante su desarrollo obtiene pequeñas recompensas que aumentan su motivación y los anima a superar el siguiente reto. 


\subsection{Las TIC y la gamificación}

Trujillo (2014, citado en Parra-González y Segura-Robles, 2019) afirma que, a través de las TIC, podemos crear artefactos digitales en los procesos de enseñanza-aprendizaje para contextualizar los mismos. De esta manera, la gamificación y, en concreto, el juego, podrían crear aprendizajes más significativos ganando espacio e importancia en educación.

En esta línea, de acuerdo con Rodríguez-Fernández (2017), las TIC han ofrecido nuevas posibilidades de gamificación en educación:

Las nuevas tecnologías han contribuido a que la gamificación evolucione, incluyendo la narrativa transmedia. El juego no tiene que desarrollarse necesariamente de manera estática, sino que permite la inclusión de dispositivos tan comunes y cotidianos como el smartphone o la tablet. Esta circunstancia abre nuevas posibilidades para atraer a aquellas generaciones acostumbradas al uso de las tecnologías (Rodríguez-Fernández, 2017, p. 182).

Corchuelo-Rodríguez (2018) señala que las herramientas TIC usadas en interacción, medición, seguimiento y control de la estrategia de gamificación facilitan y optimizan la gestión del docente.

Rodríguez-Fernández (2017) presenta una experiencia del uso de Kahoot como herramienta educativa en el ámbito universitario, en que se utiliza la aplicación para crear cuestionarios y obtener feedback de los alumnos a través de su smartphone en tiempo real en el aula. Como apunta Rodríguez-Fernández (2017), Kahoot es una herramienta de juego para realizar actividades en el aula, que permite la gamificación y la inclusión del smartphone en el aula. Además, contribuye a la mejora del aprendizaje y de la asistencia y participación del estudiante, genera dinámicas participativas en el aula y el alumnado la percibe como un juego y no como un sistema de evaluación.

\subsection{Escape room en Educación Secundaria}

El escape room o breakout edu proviene de los videojuegos en línea y se está extendiendo en las aulas. El escape room es una dinámica de aventura real ambientada normalmente en una habitación de la que se debe escapar, mientras que en breakout edu se tiene que abrir una caja. Se trata de un reto que el alumnado organizado en grupos (2-6 personas) debe superar mediante la resolución de una serie de enigmas o pruebas antes de que se acabe el tiempo establecido (45 minutos o una hora, por ejemplo). Constituye una experiencia excepcional ya que el alumnado debe dialogar, opinar y aportar para poder conseguir el objetivo de resolver la situación planteada.

Según Rondón y Moreno (2019), el juego ha demostrado ser una herramienta útil para dinamizar contenidos complejos y provocar una mayor interiorización debido a su naturaleza neuroeducativa. El profesorado consigue despertar y mantener el interés del alumnado, aumenta la motivación, se fomenta el trabajo colaborativo, se mejora la competencia comunicativa, se aplica el razonamiento deductivo y se desarrolla la inteligencia emocional y la adquisición de habilidades como la cooperación, la resiliencia y la capacidad para resolver problemas. Para el alumnado el juego le permite poder fallar sin miedo, porque siempre puede rectificar antes de llegar al final del juego. Además, se puede trabajar cualquier contenido curricular y en cualquier nivel educativo.

Desde la perspectiva del profesorado, aunque durante el juego sólo actúa como room master, es decir, persona encargada de la supervisión de la actividad (también puede facilitar alguna pista en caso de dificultad); la preparación de este trabajo supone un reto creativo y, si es exitoso, aumenta su satisfacción y motivación.

\section{PROPUESTA DIDÁCTICA}

En este artículo se describe una práctica formativa gamificada implementada en Educación Secundaria. Se trata de una propuesta didáctica, basada en un diseño original, para trabajar la literatura mediante el escape room, una de las estrategias metodológicas emergentes de mayor éxito en los últimos años que proviene de los videojuegos en línea, en la que el alumnado debe leer, dialogar, opinar y aportar para poder conseguir el objetivo de resolver la situación planteada.

Además, la gamificación se ha visto enriquecida por las aportaciones de la tecnología digital con una mayor capacidad interactiva que resulta más motivadora para el alumnado, como explicaremos a continuación.

\subsection{Contextualización}

La implementación de la propuesta didáctica se ha llevado a cabo en un centro público de Educación Secundaria de la ciudad de València. El alumnado participante estaba formado por tres grupos de $1^{\circ}$ de ESO, en total 60 alumnos. Las actividades se realizaron en el primer trimestre del curso en la asignatura de Valencià, Llengua i Literatura. 
Concretamente se trabajaron las lecturas y las actividades coincidiendo con la festividad de Todos los Santos ya que se pretendía que el alumnado conociera las leyendas relacionadas con los seres fantásticos más representativos de la cultura popular valenciana que podían dar miedo a los niños. De esta forma, se conectaban los referentes de la fiesta de difuntos que conoce actualmente el alumnado, referidos a los personajes y las historias de miedo, con los de la tradición popular.

\subsection{Objetivos}

Introduciendo la gamificación y las TIC en el aula de Lengua y Literatura de Educación Secundaria nos proponemos:

a. Utilizar el juego como recurso educativo para potenciar la educación literaria, mediante el fomento del disfrute por el aprendizaje y la motivación de los estudiantes.

b. Acceder a una mayor diversidad de textos, ampliar los recursos para reforzar diferentes habilidades lectoras y la interacción con otros lectores.

c. Mejorar la comprensión lectora y la interpretación de los textos literarios.

d. Estudiar los elementos de la narración: narrador, estructura, personajes, espacio, tiempo.

e. Facilitar la cooperación entre el alumnado, difundir sus producciones en las redes sociales.

f. Reforzar diferentes habilidades lingüísticas, la creatividad y la originalidad.

g. Normalizar la tecnología para trabajar en el aula y los recursos que favorecen la posibilidad de compartir información.

h. Desarrollar la capacidad para resolver problemas.

\subsection{Contenidos y competencias}

Mediante la propuesta didáctica que presentamos se trabajan los siguientes contenidos curriculares:

- Reconocimiento de las características propias de los géneros literarios a partir de una selección de textos en prosa.

- La narración en prosa. Elementos de las narraciones.

- Análisis sencillo de textos: localización guiada del texto en su contexto social, cultural e histórico; identificación de la época literaria; contenido de la obra o texto (tema, tópicos, argumento, personajes, estructura, formas y lenguaje literario e intención del autor/a).

- Aproximación al conocimiento de otros mundos (reales o imaginarios) a partir de la lectura.

- Lectura guiada y comprensión de textos y obras literarias, de acuerdo con el nivel del alumnado, como base para la formación de la personalidad literaria y el goce personal.

- Técnicas de elaboración de trabajos de investigación sencilla y supervisada sobre una lectura realizada.

- Fomento del hábito lector, por medio de la participación en iniciativas que impulsan la realización de lecturas amenas (club de lectores, ciberlecturas, tertulias literarias, plan lector del centro, etc.).

- Uso de un lenguaje no discriminatorio.

- Utilización guiada de las TIC en todas las fases del proceso: localizar, seleccionar, tratar, organizar y almacenar la información; crear textos escritos o audiovisuales; presentar difundir y compartir el trabajo.

- Almacenamiento de la información digital en dispositivos informáticos y servicios de la red.

Teniendo en cuenta el tipo de contenidos, el alumnado desarrolla, en la secuencia de actividades correspondiente, la competencia en comunicación lingüística (CCLI), la competencia digital (CD), la competencia de aprender a aprender (CAA) y la competencia de conciencia y expresión culturales (CEC).

\subsection{Metodología}

Utilizamos la gamificación o ludificación como estrategia motivadora para mejorar el proceso de enseñanza-aprendizaje mediante la creación de un escape room para trabajar textos literarios. El alumnado tiene que solucionar enigmas con un tiempo establecido de manera que se potencia el trabajo colaborativo, la habilidad para resolver problemas, la competencia comunicativa y el razonamiento deductivo.

La gamificación se ha visto enriquecida por las aportaciones de la tecnología digital con una mayor capacidad interactiva que resulta más motivadora para el alumnado:

- La secuencia de actividades está planteada para trabajar conjuntamente los libros y la tecnología: poner en marcha procesos de aprendizaje y de enseñanza que no serían posibles sin las TIC y está fundamentada en el constructivismo y el aprendizaje significativo.

- Tenemos en cuenta las posibilidades que hay en la red para investigar, reflexionar y compartir. 
- Supone que el lector también se transforma en autor con documentos escritos, audiovisuales, hipervínculos, etc.

- Se trata de una propuesta didáctica adaptable a cualquier contenido curricular y nivel educativo.

- Es fundamental el trabajo cooperativo y la difusión por medio de redes sociales, exposiciones en el centro, participación en concursos, etc.

En este artículo presentamos una propuesta de aula en que se aplica esta metodología que consiste en un juego dirigido, con unas reglas específicas que se explican previamente, con la finalidad de descubrir enigmas a partir de la búsqueda de información y de la lectura de las leyendas valencianas que encontramos en el libro de Víctor Labrado Llegendes valencianes (2007). En las leyendas recogidas se trabaja la tradición oral con seres fantásticos de la cultura popular valenciana: butonis, la Quarantamaula, moro Mussa, l'home del sac... Se organizan grupos que tienen que descubrir los enigmas escondidos sobre diferentes leyendas en el mínimo tiempo posible. Para ello, deben encontrar las respuestas que se les plantean en los códigos $\mathrm{QR}$, incorporados en diferentes imágenes de los protagonistas de estas leyendas (véase figura 1).

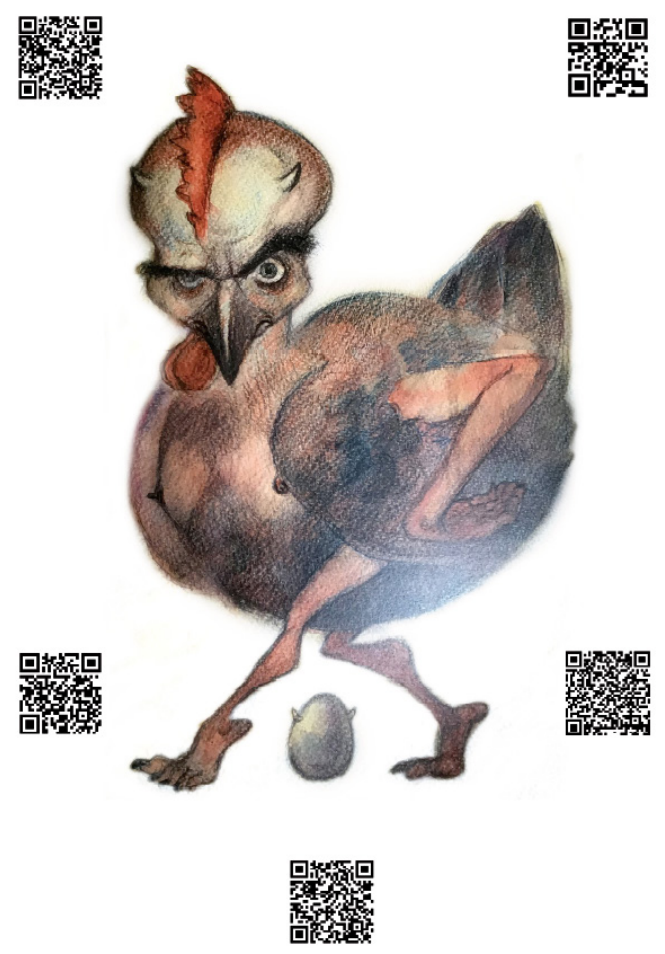

Figura 1. Imagen de uno de los personajes de las leyendas con los códigos QR.

La secuencia de actividades y las instrucciones se presentan en el blog de la materia. Los juegos están dirigidos a alumnado de Secundaria y se pueden adaptar a diferentes cursos, aunque habitualmente la lectura de leyendas tradicionales valencianas suele programarse en $1^{\circ}$ o $2^{\circ}$ de ESO.

\subsection{Actividades}

En el juego, que hemos llamado "En busca de enigmas legendarios", la secuencia de actividades se puede dividir en tres fases:

1. Actividad previa en que se visualiza un vídeo con una historia tradicional de miedo que da pie a una pregunta para introducir el relato.

2. Superación de unas pruebas que consisten en conseguir una tarjeta sobre una leyenda en la cual los participantes encontrarán la información para responder las preguntas formuladas en los códigos QR que aparecen por la sala en carteles con imágenes de los monstruos protagonistas de estas leyendas. Una vez leen la leyenda y resuelven los enigmas se tienen que enviar las respuestas con una etiqueta a Instagram.

3. Aquellos que consigan antes contestar correctamente las preguntas reciben una recompensa y pueden salir de la sala donde están encerrados.

Para conseguir la tarjeta que permitirá el acceso al juego hay que responder a la pregunta: ¿Qué te daría más miedo? ¿Un monstruo deforme, un animal peligroso o un demonio? Con esta tarjeta tienen que buscar la leyenda co- 
rrespondiente al monstruo que han elegido. Mediante la interpretación de la lectura de las leyendas han de descubrir los enigmas que se les plantean cuando escanean el código QR. Si no encuentran todas las respuestas en las leyendas, pueden consultar información en la red. También disponen de un comodín que les permite recibir ayuda con una pista por parte del profesorado.

Las respuestas se subirán a Instagram con una etiqueta de la actividad, que facilitará al profesorado el seguimiento de la misma. Finalmente, se preparará una exposición de pósteres sobre las leyendas con los personajes, la localización, la moraleja, la conexión con otras historias y las expresiones destacadas de la historia.

\subsection{Recursos}

Los juegos y las instrucciones se pueden presentar en el blog de la materia o bien en hojas o tarjetas, para que el alumnado pueda seguirlas durante el proceso. Es imprescindible que los participantes en el juego lean los textos literarios para poder interpretarlos y contestar correctamente a las preguntas y enigmas planteados. La búsqueda de información se realiza también en diferentes webs y se utiliza la red social Instagram para dar a conocer los resultados de la búsqueda, por lo tanto, se necesita poder acceder a un ordenador o un móvil.

A continuación, listamos los materiales necesarios para el desarrollo de la propuesta didáctica:

- Proyector para mostrar las diapositivas mediante las cuales se realiza la explicación del argumento.

- Cuenta atrás con el tiempo estipulado para escapar de la habitación. Para ello utilizamos un monitor externo conectado al mismo ordenador.

- Música para ambientar la escena.

- Lector de código QR.

- Ilustraciones de los personajes de las leyendas.

- Tarjetas, sobres e instrucciones del juego.

- Identificadores para los participantes con el nombre elegido para su grupo.

- Comodines para recibir ayuda.

- Póster digital.

\subsection{Evaluación}

La evaluación formativa nos permite organizar y guiar la acción educativa puesto que podemos confirmar o modificar las representaciones del alumnado. De esta manera, ajustaremos las actividades al grado de conocimientos adquiridos ya que la evaluación formativa está estrechamente vinculada a las actividades de aprendizaje de cada alumno. Tal como afirma Sanmartí (2010), encontramos dos finalidades en la evaluación: como medio para regular los aprendizajes y como medio para comprobar qué se ha aprendido.

Como indica Boluda $(2013,14)$, la evaluación formativa no tiene que tener como principal finalidad calificar al alumno/a, sino que tiene que llevarse a cabo a lo largo del proceso de enseñanza-aprendizaje para mejorarlo y, por lo tanto, tiene que conseguir estimular una actitud de observación, de recogida de datos y de análisis. Se trata de percibir lo más pronto posible las capacidades del alumnado y las disfunciones de la acción educativa. De esta manera, se consigue ayudar al profesorado a enseñar mejor, aportar información durante el proceso para mejorar los resultados finales y hacer participar el alumno/a en su proceso de formación y en la toma de decisiones.

Por otro lado, las decisiones también las deben tomar los propios aprendices, muchas veces a partir de contrastar las propias producciones con las de los compañeros y las compañeras mediante la coevaluación, tal como señalan Oliveras, Sanmartí y Simón (2018).

Además, con las herramientas digitales se pueden compartir de forma más inmediata y continuada los objetivos de aprendizaje, las revisiones y las correcciones a lo largo del proceso de forma que quede claro qué es lo que queremos que se tenga en cuenta para llegar al resultado final.

Para realizar la evaluación del trabajo se tiene en cuenta:

- La observación del proceso y el resultado de las pruebas: se valora la participación activa y el trabajo cooperativo.

- La valoración de la expresión oral y escrita.

- La información sobre autores y obras conseguida en las pruebas.

- La preparación de un cuestionario en línea con preguntas propuestas por cada grupo: lo que supone un planteamiento y una explicación de los contenidos más destacados.

Como instrumento de evaluación, proponemos la siguiente rúbrica que el profesorado puede adaptar y emplear según los diferentes textos trabajados y los acuerdos tomados con el alumnado sobre los criterios de evaluación. En esta rúbrica (tabla 1) se destaca la valoración de cuatro apartados: contenido, aspectos formales, organización del equipo de trabajo y aspectos técnicos. 
Tabla 1. Rúbrica de evaluación del juego

\begin{tabular}{|l|l|l|l|l|}
\hline CONTENIDO (40\%) & \multicolumn{1}{|c|}{ Muy adecuado } & \multicolumn{1}{|c|}{ Adecuado } & \multicolumn{1}{c|}{ Poco adecuado } & \multicolumn{1}{c|}{ Inadecuado } \\
\hline $\begin{array}{l}\text { Descubrimiento de } \\
\text { los enigmas }\end{array}$ & $\begin{array}{l}\text { Se han resuelto los } \\
\text { enigmas utilizando } \\
\text { un pensamiento } \\
\text { deductivo y } \\
\text { reflexivo y se han } \\
\text { compartido en } \\
\text { Instagram en poco } \\
\text { tiempo }\end{array}$ & $\begin{array}{l}\text { Las soluciones de } \\
\text { los enigmas son casi } \\
\text { todas correctas y } \\
\text { se han compartido } \\
\text { en Instagram en el } \\
\text { tiempo establecido }\end{array}$ & $\begin{array}{l}\text { Las soluciones de } \\
\text { los enigmas no son } \\
\text { muy correctas y } \\
\text { se han compartido } \\
\text { en Instagram en el } \\
\text { tiempo establecido }\end{array}$ & $\begin{array}{l}\text { Las enigmas no son } \\
\text { los correctas y no } \\
\text { se han compartido en } \\
\text { Instagram }\end{array}$ \\
\hline Póster de la leyenda & $\begin{array}{l}\text { La información sobre } \\
\text { la leyenda es muy } \\
\text { adecuada y } \\
\text { está presentada } \\
\text { de forma clara y } \\
\text { precisa }\end{array}$ & $\begin{array}{l}\text { La información } \\
\text { sobre la leyenda } \\
\text { es adecuada y está } \\
\text { presentada de forma } \\
\text { correcta }\end{array}$ & $\begin{array}{l}\text { La información sobre } \\
\text { la leyenda no es muy } \\
\text { completa y no se } \\
\text { entiende muy bien }\end{array}$ & $\begin{array}{l}\text { La información sobre } \\
\text { la leyenda no está } \\
\text { completa y no se } \\
\text { entiende }\end{array}$ \\
\hline $\begin{array}{l}\text { Riqueza de fuentes } \\
\text { de información } \\
\text { utilizadas }\end{array}$ & $\begin{array}{l}\text { La información } \\
\text { expuesta es muy } \\
\text { relevante }\end{array}$ & $\begin{array}{l}\text { Los conceptos } \\
\text { expuestos son } \\
\text { relevantes }\end{array}$ & $\begin{array}{l}\text { No todos los } \\
\text { conceptos expuestos } \\
\text { son muy relevantes }\end{array}$ & $\begin{array}{l}\text { Los conceptos } \\
\text { expuestos tienen } \\
\text { una relación lejana } \\
\text { con el foco del } \\
\text { trabajo }\end{array}$ \\
\hline
\end{tabular}

\begin{tabular}{|c|c|c|c|c|}
\hline $\begin{array}{c}\text { ASPECTOS } \\
\text { FORMALES }(30 \%)\end{array}$ & Muy adecuado & Adecuado & Poco adecuado & Inadecuado \\
\hline $\begin{array}{l}\text { Expresión oral y } \\
\text { escrita }\end{array}$ & $\begin{array}{l}\text { La articulación y la } \\
\text { expresión es clara y } \\
\text { correcta }\end{array}$ & $\begin{array}{l}\text { La articulación y } \\
\text { la expresión es } \\
\text { bastante clara y } \\
\text { correcta }\end{array}$ & $\begin{array}{l}\text { La articulación y la } \\
\text { expresión es poco } \\
\text { clara y correcta }\end{array}$ & $\begin{array}{l}\text { La articulación y la } \\
\text { expresión no resulta } \\
\text { clara ni coherente }\end{array}$ \\
\hline Normativa y registro & $\begin{array}{l}\text { El trabajo muestra } \\
\text { corrección gramatical. } \\
\text { Vocabulario amplio y } \\
\text { preciso. } \\
\text { Aspectos } \\
\text { morfosintácticos, } \\
\text { de concordancia, } \\
\text { ortografía y registro } \\
\text { adecuados }\end{array}$ & $\begin{array}{l}\text { Se observan errores } \\
\text { gramaticales } \\
\text { puntuales. El trabajo } \\
\text { es adecuado al } \\
\text { registro académico, } \\
\text { con alguna desviación } \\
\text { del estándar y del } \\
\text { registro formal }\end{array}$ & $\begin{array}{l}\text { Hay errores que } \\
\text { muestran un } \\
\text { desconocimiento } \\
\text { de la normativa } \\
\text { ortográfica y } \\
\text { gramatical. El trabajo } \\
\text { es adecuado al } \\
\text { ámbito académico, } \\
\text { pero presenta } \\
\text { coloquialismos }\end{array}$ & $\begin{array}{l}\text { El trabajo muestra } \\
\text { una expresión llena } \\
\text { de errores } \\
\text { ortográficos y } \\
\text { gramaticales. El } \\
\text { registro empleado } \\
\text { no es adecuado al } \\
\text { ámbito académico. } \\
\text { Uso abundante } \\
\text { de expresiones } \\
\text { coloquiales }\end{array}$ \\
\hline $\begin{array}{l}\text { Propiedades textuales } \\
\text { (adecuación, } \\
\text { coherencia y } \\
\text { cohesión) }\end{array}$ & $\begin{array}{l}\text { Muestra coherencia } \\
\text { en el tratamiento y } \\
\text { estructuración de } \\
\text { la información, y } \\
\text { cohesión en aspectos } \\
\text { como el orden, } \\
\text { las pausas y los } \\
\text { conectores. } \\
\text { Se consigue el } \\
\text { propósito que se pedía }\end{array}$ & $\begin{array}{l}\text { El trabajo podría tener } \\
\text { una mayor coherencia } \\
\text { y cohesión, con } \\
\text { una estructuración } \\
\text { de las ideas más } \\
\text { ordenada y clara. En } \\
\text { algún momento hay } \\
\text { alguna redundancia } \\
\text { no justificable, } \\
\text { algún implícito } \\
\text { no recuperable o } \\
\text { ambigüedades. } \\
\text { Se consigue el } \\
\text { propósito }\end{array}$ & $\begin{array}{l}\text { Hay una falta de } \\
\text { orden y de uso } \\
\text { de conectores. } \\
\text { Presentación confusa } \\
\text { que dificulta la } \\
\text { comprensión: } \\
\text { alguna confusión, } \\
\text { imprecisión o } \\
\text { ambigüedad. } \\
\text { Se aleja de las } \\
\text { convenciones } \\
\text { prototípicas. } \\
\text { Presenta errores } \\
\text { graves en la } \\
\text { cohesión. } \\
\text { Se consigue sólo en } \\
\text { parte el propósito }\end{array}$ & $\begin{array}{l}\text { No se respetan } \\
\text { la coherencia y } \\
\text { cohesión. } \\
\text { Presenta deficiencias } \\
\text { notables que } \\
\text { dificultan su } \\
\text { comprensión: hay } \\
\text { incongruencias, } \\
\text { ideas poco } \\
\text { desarrolladas, etc. } \\
\text { Tiene muchos errores } \\
\text { de cohesión. } \\
\text { No se consigue el } \\
\text { propósito que se pedía }\end{array}$ \\
\hline
\end{tabular}




\begin{tabular}{|c|c|c|c|c|}
\hline $\begin{array}{l}\text { ORGANIZACIÓN } \\
\text { DEL EQUIPO DE } \\
\text { TRABAJO (20\%) }\end{array}$ & Muy adecuado & Adecuado & Poco adecuado & Inadecuado \\
\hline $\begin{array}{l}\text { Participación, } \\
\text { organización y } \\
\text { atención }\end{array}$ & $\begin{array}{l}\text { Espíritu colaborador } \\
\text { y participación activa } \\
\text { en el grupo y en la } \\
\text { realización de las } \\
\text { actividades. } \\
\text { En la visualización } \\
\text { del trabajo de los } \\
\text { compañeros ha } \\
\text { prestado atención y ha } \\
\text { demostrado capacidad } \\
\text { de análisis }\end{array}$ & $\begin{array}{l}\text { Espíritu colaborador } \\
\text { y participación } \\
\text { no muy activa en } \\
\text { el grupo y en la } \\
\text { realización de las } \\
\text { actividades. } \\
\text { En la visualización } \\
\text { del trabajo de } \\
\text { los compañeros } \\
\text { ha prestado poca } \\
\text { atención y ha } \\
\text { demostrado escasa } \\
\text { capacidad de } \\
\text { análisis }\end{array}$ & $\begin{array}{l}\text { Poco espíritu } \\
\text { colaborador y } \\
\text { participación no } \\
\text { muy activa en } \\
\text { el grupo y en la } \\
\text { realización de las } \\
\text { actividades. } \\
\text { En la visualización } \\
\text { del trabajo de los } \\
\text { compañeros ha } \\
\text { prestado muy } \\
\text { poca atención y } \\
\text { no ha demostrado } \\
\text { mucha capacidad de } \\
\text { análisis }\end{array}$ & $\begin{array}{l}\text { Falta de espíritu } \\
\text { colaborador y de } \\
\text { participación activa } \\
\text { en el grupo y en la } \\
\text { realización de las } \\
\text { actividades. } \\
\text { En la visualización } \\
\text { del trabajo de los } \\
\text { compañeros ha } \\
\text { prestado atención y } \\
\text { no ha demostrado } \\
\text { capacidad de análisis }\end{array}$ \\
\hline $\begin{array}{l}\text { Distribución de } \\
\text { tareas }\end{array}$ & $\begin{array}{l}\text { Se reparten las } \\
\text { tareas en función } \\
\text { de la competencia y } \\
\text { motivación de cada } \\
\text { miembro del equipo, } \\
\text { teniendo en cuenta } \\
\text { las condiciones } \\
\text { necesarias para } \\
\text { alcanzar un } \\
\text { resultado final de } \\
\text { calidad }\end{array}$ & $\begin{array}{l}\text { Se reparten las tareas } \\
\text { de forma equilibrada, } \\
\text { valorando cuál es la } \\
\text { mejor aportación que } \\
\text { cada miembro del } \\
\text { equipo puede hacer } \\
\text { para la consecución } \\
\text { de los objetivos } \\
\text { comunes }\end{array}$ & $\begin{array}{l}\text { Se reparten las } \\
\text { tareas considerando } \\
\text { únicamente los } \\
\text { objetivos a conseguir, } \\
\text { sin tener en cuenta } \\
\text { la competencia y la } \\
\text { motivación individual } \\
\text { de cada miembro del } \\
\text { equipo }\end{array}$ & $\begin{array}{l}\text { Se reparten las tareas } \\
\text { aleatoriamente, sin } \\
\text { tener en cuenta ni los } \\
\text { objetivos a conseguir } \\
\text { ni la competencia y } \\
\text { motivación de los } \\
\text { miembros del equipo }\end{array}$ \\
\hline
\end{tabular}

\begin{tabular}{|l|l|l|l|l|}
\hline \multicolumn{1}{|c|}{$\begin{array}{c}\text { ASPECTOS } \\
\text { TÉCNICOS (10\%) }\end{array}$} & \multicolumn{1}{|c|}{ Muy adecuado } & \multicolumn{1}{c|}{ Adecuado } & \multicolumn{1}{c|}{ Poco adecuado } & \multicolumn{1}{c|}{ Inadecuado } \\
\hline $\begin{array}{l}\text { Materiales y } \\
\text { recursos utilizados }\end{array}$ & $\begin{array}{l}\text { Todos los materiales } \\
\text { y recursos utilizados } \\
\text { son los propios del } \\
\text { trabajo planteado } \\
\text { y se justifican } \\
\text { adecuadamente }\end{array}$ & $\begin{array}{l}\text { La mayoría de los } \\
\text { materiales y } \\
\text { recursos utilizados } \\
\text { son los indicados } \\
\text { en el trabajo } \\
\text { planteado ye } \\
\text { justifican } \\
\text { adecuadamente }\end{array}$ & $\begin{array}{l}\text { Solo algunos de los } \\
\text { materiales y recursos } \\
\text { utilizados son los } \\
\text { propios del trabajo } \\
\text { planteado y se } \\
\text { justifican } \\
\text { adecuadamente }\end{array}$ & $\begin{array}{l}\text { Ninguno de los } \\
\text { materiales y recursos } \\
\text { utilizados son los } \\
\text { propios del trabajo } \\
\text { planteado y no se } \\
\text { justifican } \\
\text { adecuadamente }\end{array}$ \\
\hline Difusión & $\begin{array}{l}\text { Se ha compartido } \\
\text { el trabajo en una } \\
\text { exposición de forma } \\
\text { efectiva }\end{array}$ & $\begin{array}{l}\text { Se ha compartido } \\
\text { el trabajo en una } \\
\text { exposición bastante } \\
\text { correctamente }\end{array}$ & $\begin{array}{l}\text { No se ha compartido } \\
\text { el trabajo de forma } \\
\text { efectiva }\end{array}$ & $\begin{array}{l}\text { No se ha c } \\
\text { ompartido la } \\
\text { información elaborada } \\
\text { durante el trabajo }\end{array}$ \\
\hline
\end{tabular}

\section{RESULTADOS}

A partir de la evaluación obtenida mediante la rúbrica y las producciones realizadas por el alumnado, se ha podido determinar hasta qué punto la introducción de la gamificación y los recursos tecnológicos pueden potenciar la educación literaria mediante la interpretación y el análisis de los textos leídos.

Sobre los contenidos propuestos respecto al mensaje y las características de las leyendas, todos los grupos participantes consiguieron resolver los enigmas a partir de la lectura y la búsqueda de información en webs recomendadas. Además, la información elaborada se compartió en Instagram y se presentó en clase de forma correcta. Después se realizó una exposición con carteles sobre los principales miedos que tenía el alumnado en la actualidad con el objetivo de reflexionar sobre los motivos y la manera de poder superarlos. Finalmente, se publicó en el periódico del Instituto una noticia con los carteles realizados (véase figura 2), las conclusiones del debate sobre la manera de ahuyentar los miedos (véase figura 3) y otras leyendas de miedo seleccionadas por el alumnado (véase figura 4). Así se propuso un principio terapéutico para vencer el miedo: enfrentarnos a él para comprender que no es para tanto. Escuchar, narrar, leer y escribir historias resulta un buen conjuro contra el miedo. 


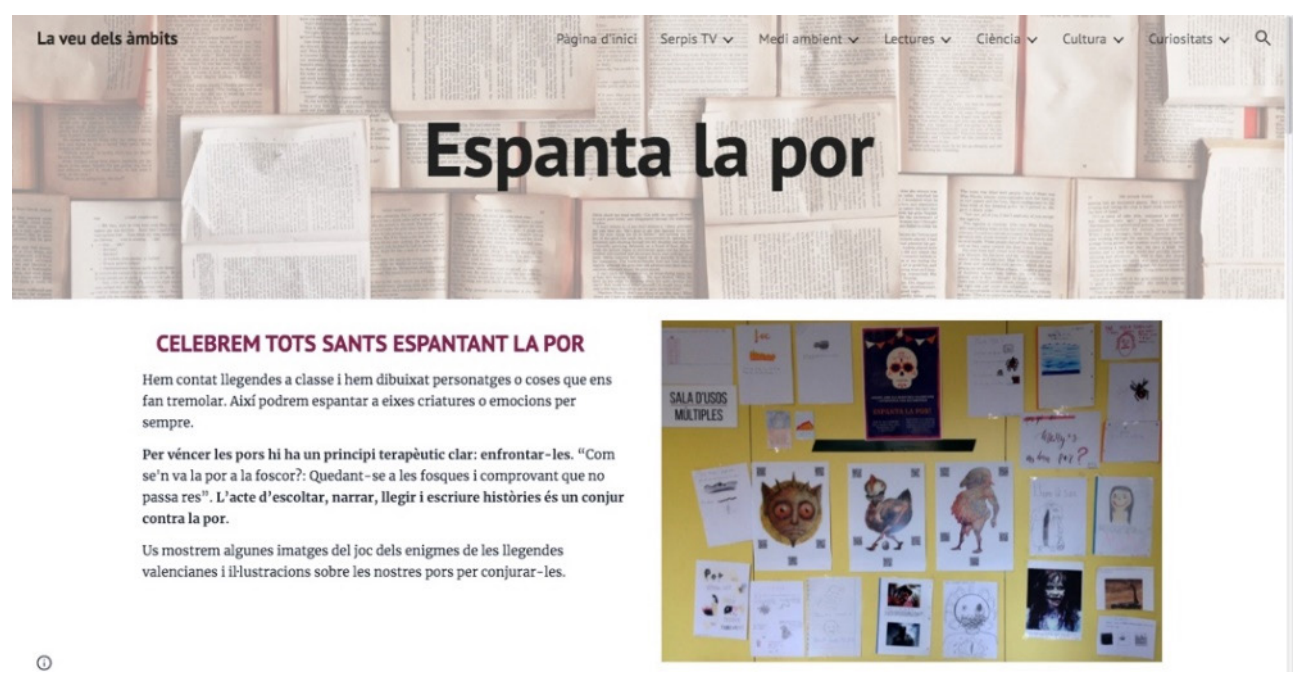

Figura 2. Noticia publicada en el periódico del Instituto sobre la exposición con los monstruos de las leyendas.
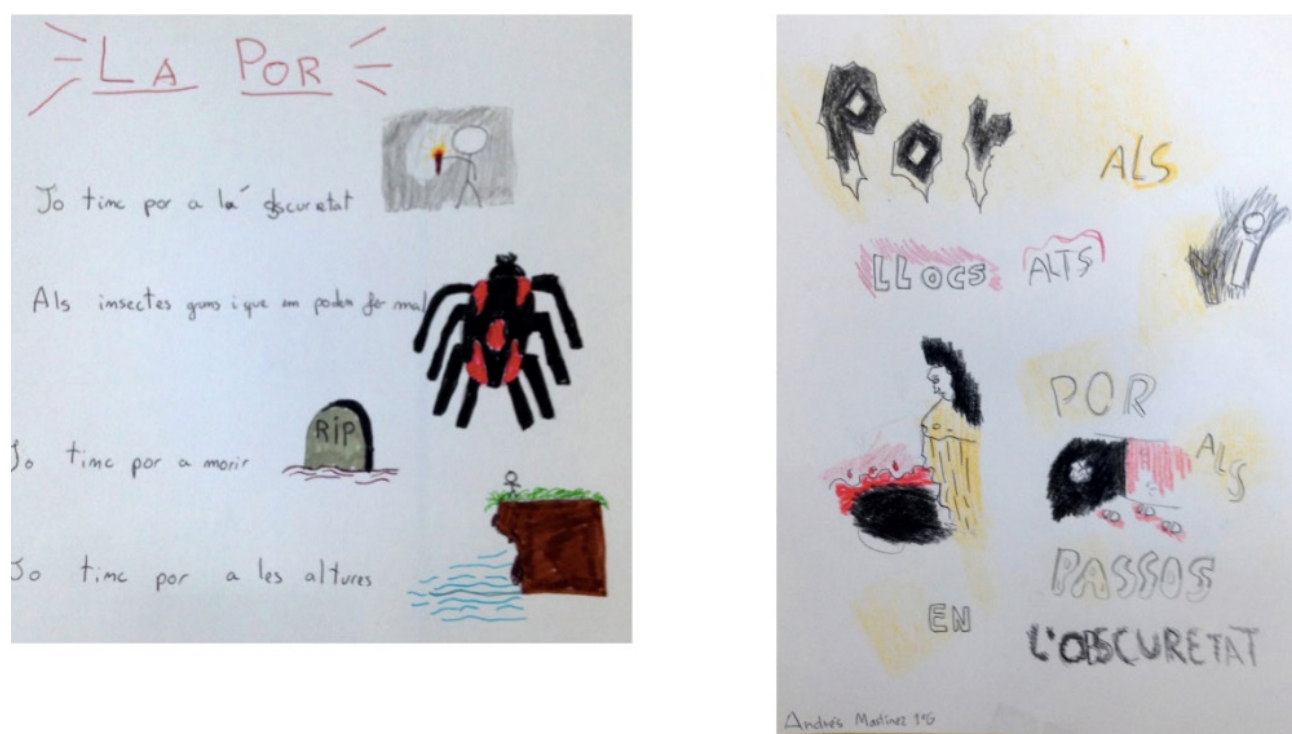

Figura 3. Carteles de la exposición sobre los miedos que hay que ahuyentar.

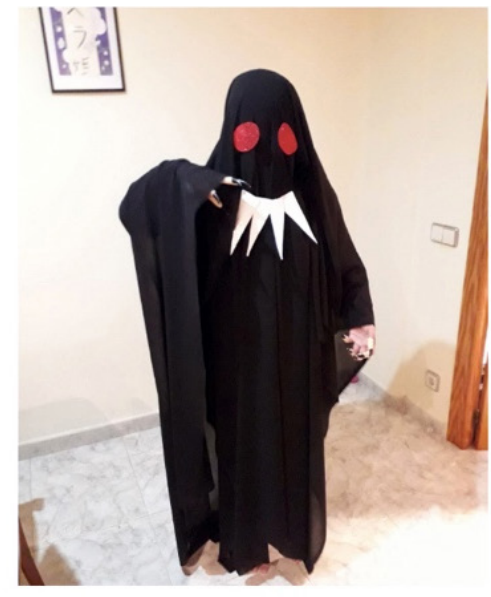

Ha passat quasi un mes des de Tots Sants però encara ens fem ressò d'aquell dia! Hui afegim una rondalla que va recollir per escrit Enric Valor que porta per títol I queixalets també! i una disfressa casolana de Queixalets feta per Tesa Montoro de ir ESOF.
I QUEIXALETS TAMBÉ!

Un cas que va passar en la vila de Penàguila. Un cas gros i esgarrifador, per cert.

Cal dir-vos de bestreta, que aquesta vila és un racó de món. Un bell racó, això sí, enmig d'intricades, ombrenques i encinglerades muntanyes. De vora el poble, davalla una costera vorejada d'oms gegantins $\mathrm{i}$ frondosos, $\mathrm{i}$ al capdavall hi ha una font abundantíssima que trona pels seus vint-i-tants canonets de bronze I el llavador, on fan les dones la bugada De dia hi ha grans rastres de dones llavant; però les nits, d'ençà

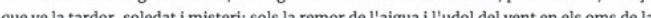
baixada.

- No aneu de nit a llavar a la font! - aconsellen les mares a les filles.

Perquè una volta, quan era joveneta, una nit de desembre que hi havia més d'un pam de neu gelada i relliscosa, Toneta la de les Alcoies no va creure sa mare i va voler anar-hi a rentar una flassada $i$ un llençol.

Eren les deu de la nit; tot era fosc com la gola de llop, i, quan va eixir del poble, encara queia alguna volveta de neu. A l'arribar al primer om de la baixada, li semblà que, entremig de la fressa que feia el vent en les branques despullades, sentia gemegar un infant.

-Això és un xiquet que plora per ací! -va dir-se tota estranyada

- Eeeeeh, Eeeeeh! - s'oïa en la fosca.

Sí que plorava un criançó, i ben prop.

Davall a pleret entre els oms. Se'l va sentir a la vora.

- Eeeeh, eeeeh!

A l'esmortida claredat d'un fanal d'oli penjat a mitjan costera, va entreveure, a la soca

Figura 4. Leyenda de miedo seleccionada por el alumnado y un disfraz de uno de los personajes protagonistas. 
Respecto a la evaluación de la expresión oral y escrita, se pudo realizar un seguimiento del proceso de corrección seguido durante las diversas actividades ya que se valoró la presentación oral y escrita del resultado de la investigación sobre los enigmas planteados, el debate sobre el mensaje que transmiten las leyendas y la manera de superar los miedos, la elaboración de carteles y de una noticia para el periódico del centro. Como los trabajos realizados formarían parte de una exposición pública en el centro y también serían compartidos en las redes sociales, debían de revisarse y corregirse para obtener una buena presentación, lo que comportaba una reflexión metalingüística en relación a las dudas gramaticales y las propiedades textuales.

Por otro lado, la secuencia de actividades supuso potenciar el trabajo colaborativo ya que se formaron grupos para participar en el juego y elaborar las propuestas de las diferentes actividades. Se siguió el criterio de repartir las tareas en función de la competencia y interés de cada miembro del grupo, por lo que también se valoró el trabajo personal de cada componente.

Finalmente, se compartió y difundió el resultado de los enigmas descubiertos en las leyendas y los carteles elaborados en el periódico del Instituto, la web y las redes sociales. De esta forma, se motivó a los estudiantes a completar y presentar su trabajo con un diseño creativo y una revisión lingüística detallada, puesto que se tenía que exponer públicamente.

A partir de estos resultados, consideramos que la gamificación junto con la utilización de recursos digitales aporta un aprendizaje significativo en este caso, ya que ha facilitado al alumnado la comprensión de textos literarios clásicos y ha potenciado el trabajo colaborativo. Mediante esta propuesta didáctica destacamos además que:

- Hemos comprobado que el escape room ha potenciado que el alumnado lea, dialogue, opine y aporte información para conseguir el objetivo de resolver la situación planteada. De manera que esta metodología participativa colabora con la interiorización de los contenidos.

- El uso de las tecnologías con la ludificación favorece la formación literaria ya que supone la posibilidad de trabajar con gran diversidad de textos, un refuerzo de las diferentes habilidades lingüísticas y la posibilidad de interactuar con otros lectores.

- La incorporación de las TIC, en este caso mediante códigos QR, cuestionario en línea, Instagram, póster digital y el blog de la materia, permite trabajar la competencia digital para favorecer la competencia lingüística y literaria. Además, la difusión en las redes sociales impulsa la creatividad y la originalidad de las producciones literarias realizadas en el aula.

\section{CONCLUSIONES}

Con la realización de esta experiencia didáctica podemos aportar evidencias sobre los beneficios de la gamificación en la formación del alumnado. La participación de equipos organizados para resolver los enigmas de las leyendas ha resultado una interesante y eficaz estrategia docente para potenciar la implicación de los discentes, alcanzar un aprendizaje más significativo mediante el trabajo colaborativo y la difusión de las producciones en las redes sociales.

La gamificación educativa facilita la motivación del alumnado al tener en cuenta los rasgos culturales de los postmillennials o Generación Z, cuyo proceso de aprendizaje se puede favorecer mediante actividades que incorporen recursos digitales. Por otra parte, esta experiencia ha exigido al docente activar su imaginación y creatividad para diseñar escenarios de aprendizaje atractivos, adoptando las mecánicas propias de los juegos para dinamizar el proceso de enseñanza-aprendizaje, combinándolas con el uso de herramientas digitales.

Mediante esta metodología se puede mejorar la comprensión lectora de los textos literarios, ya que se requiere una lectura atenta e interpretativa para que puedan resolverse los enigmas propuestos sobre las leyendas. Además, los participantes han de localizar los elementos de la narración, así como desarrollar la capacidad para resolver problemas conectados con estos elementos de carácter literario. La búsqueda de otras obras, personajes y tópicos de la literatura clásica permite que el alumnado demuestre sus referentes literarios y que aprenda a seleccionar la información que necesita de la red.

Finalmente, la multimodalidad de esta actividad gamificada supone trabajar diferentes habilidades lingüísticas, la expresión oral y escrita, potenciar la creatividad audiovisual y la originalidad en la elaboración del producto final mediante la creación de un póster digital sobre la leyenda trabajada.

Podemos afirmar que con este tipo de propuestas se consigue normalizar la tecnología incorporándola como un recurso didáctico en el aula. Además, la gamificación resulta motivadora y refuerza la participación y la comprensión lectora.

\section{REFERENCIAS BIBLIOGRÁFICAS}

Boluda, Gemma (2013). De què parlem quan ens referim a l'avaluació formativa. L'avaluació formativa a la universitat des de la perspectiva docent: visions i experiències a la Universitat de Vic. Vic: Universitat de Vic. Https://www.mdx. cat/bitstream/handle/10854/2676/artconlli_a2013_boluda_gemma_parlem_quan.pdf?sequence=1\&isAllowed=y.

Csikszentmihalyi, Mihaly (1975). Beyond Boredom and Anxiety: Experiencing Flow in Work and Play. San Francisco: Jossey-Bass. 
Csikszentmihalyi Mihaly (1990). Flow: The Psychology of Optimal Experience. New York: Haper and Row. Https:// archive.org/details/flowpsychologyof00csik.

Contreras, Ruth Sofía (2016). Elementos de juego y motivación: reflexiones entorno a una experiencia que utiliza gamificación en una asignatura de grado para Game Designers. En Ruth S. Contreras y José Luis Eguia (eds.), Gamificación en aulas Universitarias (p. 55-66). Bellaterra: Institut de la Comunicació, Universitat Autònoma de Barcelona.

Contreras, Ruth Sofía (2017). Gamificación en escenarios educativos. Revisando literatura para aclarar conceptos. En Ruth S. Contreras y José Luis Eguia (eds.). Experiencias de gamificación en aulas (p. 11-17). Bellaterra: Institut de la Comunicació, Universitat Autònoma de Barcelona.

Contreras, Ruth Sofía y José Luis Eguia (eds.) (2016). Gamificación en aulas universitarias. Bellaterra: Institut de la Comunicació, Universitat Autònoma de Barcelona. Https://ddd.uab.cat/pub/llibres/2016/166455/Ebook_INCOMUAB_10.pdf.

Contreras, Ruth S. y José Luis Eguia (eds.) (2017). Experiencias de gamificación en aulas. Bellaterra: Institut de la Comunicació, Universitat Autònoma de Barcelona. Https://ddd.uab.cat/pub/llibres/2018/188188/ebook15.pdf.

Corchuelo-Rodríguez, C. A. (2018). Gamificación en educación superior: experiencia innovadora para motivar estudiantes y dinamizar contenidos en el aula. Edutec. Revista Electrónica de Tecnología Educativa, 63, 29-41. Https://doi. org/10.21556/edutec.2018.63.927.

Deterding, Sebastian, Dan Dixon, Rilla Khaled y Lennart E. Nacke (2011). Gamification: Toward a Definition. En Proceedings of the 2011 Workshop Gamification: Using Game Design Elements in Non-Game Contexts. Nueva York: ACM. http:/gamification-research.org/wp-content/uploads/2011/04/02-Deterding-Khaled-Nacke-Dixon.pdf

Hamari, Juho, Jonna Koivisto y Harri Sarsa (2014). Does Gamification Work?: A Literature Review of Empirical Studies on Gamification. Hawaii (USA): 47th Hawaii Int. Conference on System Sciences.

Labrado, Víctor (2007). Llegendes valencianes: criatures mítiques de la tradició oral. Alzira: Bromera.

Marín, Inma (2014 abril 4). Es pot gamificar l'educació? El Diari de l'Educació. Http://diarieducacio.cat/blogs/ bofill/2014/04/04/es-pot-gamificar-leducacio/.

Marín, Verónica (2015). La gamificación educativa. Una alternativa para la enseñanza creativa. Digital Education Journal, 90, 1-4. Http://revistes.ub.edu/index.php/der/article/view/13433/pdf.

Oliveras, Begonya, Neus Sanmartí y Marta Simón (2018). Retos de la educación secundaria actual. Aula de Secundaria, 25, 29-32.

Ortiz-Colón, Ana María, Juan Jordán y Míriam Agredal (2018). Gamificación en educación: una panorámica sobre el estado de la cuestión. Educação e Pesquisa: Revista da Faculdade de Educação da Universidade de São Paulo, 44. Https://doi.org/10.1590/s1678-4634201844173773.

Pajuelo, L. (2018 agosto 28) Escape room en educación: la tendencia que arrasa en las aulas. Educación 3.0, 30 , 16-24. Https://www.educaciontrespuntocero.com/noticias/escape-room-en-educacion/.

Parente, Daniel (2016). Gamificación en la educación. En Ruth S. Contreras y José Luis Eguia (eds.). Gamificación en aulas Universitarias (p. 11-21). Bellaterra: Institut de la Comunicació, Universitat Autònoma de Barcelona.

Parra-González, Elena y Adrián Segura-Robles (2019). Producción científica sobre gamificación en educación: un análisis cienciométrico. Revista de Educación, 386, 113-135. Https://doi.org/10.4438/1988-592X-RE-2019-386-429.

Paula, Óscar de (2017). Reader Wars: motivar a los alumnos por la lectura. En Ruth S. Contreras y José Luis Eguia (eds.). Experiencias de gamificación en aulas (p. 79-94). Bellaterra: Institut de la Comunicació, Universitat Autònoma de Barcelona.

Piaget, Jean (1961). La formación del símbolo en el niño: imitación, juego y sueño. Imagen y representación. México: Fondo de Cultura Económica.

Ramírez, José Luis (2014). Gamificación. Mecánicas de juegos en tu vida personal y profesional. Madrid: Servicio Comercial del Libro.

Ríos, Araceli, Yolanda Muñoz, Pilar Castro y José Luis Arroyo (2019). Gamificación, estrategia compartida entre universidad, empresa y millennials. REDU. Revista de Docencia Universitaria, 17(2), 73-88. Https://doi.org/10.4995/ redu.2019.11479.

Ripoll, O. (2016). Taller de creació de jocs, una asignatura gamificada. En Ruth S. Contreras y José Luis Eguia (eds.). Gamificación en aulas Universitarias (p. 25-37). Bellaterra: Institut de la Comunicació, Universitat Autònoma de Barcelona.

Rodríguez-Fernández, Leticia (2017). Smartphones y aprendizaje: el uso de Kahoot en el aula universitaria. Revista Mediterránea de Comunicación, 8(1), 181-190. Https://doi.org/10.14198/MEDCOM2017.8.1.13.

Rondón García, Marina y Antonio José Moreno Guerrero (2019). El fomento de la lectura a través del escape room. Textos de Didáctica de la Lengua y la Literatura, 85, 62-67.

Sanmartí, Neus (2010). Avaluar per aprendre. L'avaluació per a millorar els aprenentatges de l'alumnat en el marc del currículum per competències. Barcelona: Generalitat de Catalunya, Departament d'Educació. Http://xtec.gencat. cat/web/.content/alfresco/d/d/workspace/SpacesStore/0024/fc53024f-626e-423b-877a-932148c56075/avaluar_per_ aprendre.pdf.

Teixes, Ferran (2015). Gamificación. Motivar jugando. Barcelona: Editorial UOC.

Vigotsky, Lev Semiónovich (1933, 1966). El papel del juego en el desarrollo. En Lev S. Vigotsky (2012). El desarrollo de los procesos psicológicos superiores. Barcelona: Crítica. 